OPEN ACCESS

Edited by:

Jing-Yan Han,

Peking University, China

Reviewed by:

Xuemei Qin,

Shanxi University, China

Jie Wang,

Guang'anmen Hospital, Academy

of Chinese Medical Sciences, China

*Correspondence:

Dong-Sheng Wang

wdsh666@126.com

Specialty section:

This article was submitted to

Vascular Physiology,

a section of the journal

Frontiers in Physiology

Received: 10 April 2019

Accepted: 12 August 2019

Published: 04 September 2019

Citation:

Zhao L-L, Qiu X-J, Wang W-B, Li R-M and Wang D-S (2019) NMR

Metabolomics and Random Forests

Models to Identify Potential Plasma Biomarkers of Blood Stasis Syndrome

With Coronary Heart Disease

Patients. Front. Physiol. 10:1109.

doi: 10.3389/fphys.2019.01109

\section{NMR Metabolomics and Random Forests Models to Identify Potential Plasma Biomarkers of Blood Stasis Syndrome With Coronary Heart Disease Patients}

\author{
Lin-Lin Zhao', Xin-Jian Qiu'², Wen-Bo Wang'2, Ruo-Meng Li3 and Dong-Sheng Wang ${ }^{2 *}$ \\ ${ }^{1}$ Health Management Department, The Third Xiangya Hospital, Central South University, Changsha, China, ${ }^{2}$ Institute \\ of Integrated Traditional Chinese and Western Medicine, Xiangya Hospital, Central South University, Changsha, China, \\ ${ }^{3}$ Traditional Chinese Medicine Department, The Third Xiangya Hospital, Central South University, Changsha, China
}

Background: Coronary heart disease (CHD) remains highly prevalent and is one of the largest causes of death worldwide. Blood stasis syndrome (BSS) is the main syndrome associated with CHD. However, the underlying biological basis of BSS with CHD is not yet been fully understood.

Materials and Methods: We proposed a metabolomics method based on ${ }^{1} \mathrm{H}-\mathrm{NMR}$ and random forest (RF) models to elucidate the underlying biological basis of BSS with CHD. Firstly, 58 cases of CHD patients, including 27 BSS and 31 phlegm syndrome (PS), and 26 volunteers were recruited from Xiangya Hospital affiliated to Central South University. A $1 \mathrm{~mL}$ venous blood sample was collected for NMR analysis. Secondly, principal component analysis (PCA), partial least squares discrimination analysis (PLS-DA) and RF was applied to observe the classification of each group, respectively. Finally, RF and multidimensional scaling (MDS) were utilized to discover the plasma potential biomarkers in CHD patients and CHD-BSS patients.

Results: The models constructed by RF could visually discriminate BSS from PS in CHD patients. Simultaneously, we obtained 12 characteristic metabolites, including lysine, glutamine, taurine, tyrosine, phenylalanine, histidine, lipid, citrate, choline, lactate, $\alpha$-glucose, $\beta$-glucose related to the CHD patients, and Choline, $\beta$-glucose, $\alpha$-glucose and tyrosine were considered as potential biomarkers of CHD-BSS.

Conclusion: The combining of ${ }^{1} \mathrm{H}-\mathrm{NMR}$ profiling with RF models was a useful approach to analyze complex metabolomics data (should be deleted). Choline, $\beta$-glucose, $\alpha$-glucose and tyrosine were considered as potential biomarkers of CHD-BSS.

Keywords: coronary heart disease, blood stasis syndrome, metabolomics, random forests, ZHENG types, Systems Biology 


\section{BACKGROUND}

Coronary heart disease (CHD) is always associated with metabolic disorder, and the metabolic risk factors, such as hyperlipidemia and diabetes, powerfully predict the cardiac events (Lacoste et al., 1995; Ansar et al., 2011). CHD remains highly prevalent and is one of the largest causes of death worldwide. Specific and sensitive diagnostic methods are critical both for early detection and treatment of CHD (Rubin, 2013). Metabonomics technologies have been studied to diagnose the presence and severity of CHD (Brindle et al., 2002). It is a newly developed platform of systems biology that allows holistic, quantitative and qualitative determination of low molecular endogenous metabolites in biofluids or tissues (Lindon et al., 2004). It concerns the dynamic multivariate metabolic changes in response to pathophysiological stimuli, genetic modification, environmental influences or drug perturbations (Nicholson and Lindon, 2008). Analysis of differential metabolites enables us to obtain novel biomarkers discovery and disturbed metabolic pathways (Sabatine et al., 2005).

Nowadays, Traditional Chinese Medicine (TCM) has become popular worldwide. Systematic, holistic and dynamic characteristics of TCM theory are perfectly coincident with metabonomics (Wang et al., 2005; Sun et al., 2012). Metabonomics technologies have been applied in study on the essence of the syndrome (ZHENG type or pattern in TCM) and shown the superiority and advanced nature. According to TCM theory, a syndrome is a combination of clinical manifestations including symptoms, signs, tongue appearances and pulse feelings. Syndrome is not only the core of TCM theory, but also the base of definite diagnosis and effective therapies ( $\mathrm{Xu}$ and Chen, 2008). It will not only improve the validity and reliability of "syndrome differentiation" through syndrome model established based on metabonomics, but also help to establish the clinical curative criteria.

Blood stasis syndrome (BSS) is the most common syndrome associated with CHD. Our previous study has shown that the metabonomics approach based on liquid chromatography/ quadrupole time-of-flight mass spectrometry (LC-Q-TOF/MS) was useful for interpreting the differentiation of syndrome [phlegm syndrome (PS) and BSS] in TCM (Zhao et al., 2014). There were 18 differential metabolites mainly involved in amino acid metabolism, purine metabolism and pyrimidine metabolism contributing to the clustering and discrimination between PS and BSS in CHD patients. However, it is clear that the two metabonomics technologies are complementary, giving information on different sets of biomarkers and providing more comprehensive classification and biomarker information.

Random forests (RFs) is machine learning algorithm which uses an ensemble of classification trees and is an ideal method for classification and feature selection. Spectral buckets are employed as input variables. RF could be employed for both supervised (outcome labels are used) and unsupervised (outcome labels are not used) learning (Breiman, 2001). In this study, we proposed a metabolomics method based on ${ }^{1} \mathrm{H}-\mathrm{NMR}$ and RF models to validate the separated trend between BSS and PS in CHD patients and acquire more potential biomarkers of BSS in CHD patients.

\section{MATERIALS AND METHODS}

\section{Subjects Collection}

A total of 58 cases CHD patients (27 BSS and 31 PS) and 26 volunteers were derived from the Xiangya Hospital affiliated to the Central South University in Hunan Province, China (June 1, 2013 to April 30, 2014). All selected CHD patients were diagnosed and confirmed by coronary angiography. Diagnosis standard of CHD refers to "nomenclature and diagnosis criteria of ischemic heart disease" which is established by the Joint International Society and Federation of Cardiology/World Health Organization Task Force on Standardization of Clinical Nomenclature (Nomenclature and criteria for diagnosis of ischemic heart disease, 1979). The syndrome was identified by three chief physicians, according to "criteria for TCM syndrome differentiation of patients with coronary heart disease" (Subcommittee of Cardiovascular Diseases of China Society of Integrated Traditional Chinese, and Western Medicine, 1991).

Patients who suffered from diabetic cardiomyopathy, hyperthyroid heart disease, hypertensive heart disease, pulmonary heart disease, anemic heart disease, systemics scleroderma heart disease, inborn coronary abnormity and rheumatic heart disease, who suffered from severe hypertension, malignant tumor, renal failure, thyroid disease, pulmonary infection, who suffered from infectious diseases, who suffered from invigorative system disease and women in pregnant or in lactation were excluded.

All patients aged from 45 to 75 were eligible for enrollment. The age and sex of volunteers in control group were groupmatched with the cases group. The study was approved by the hospital ethics committee and all subjects provided written informed consent.

\section{Chemicals and Reagents}

$\mathrm{NaCl}, \mathrm{K}_{2} \mathrm{HPO}_{4} \cdot 3 \mathrm{H}_{2} \mathrm{O}$, and $\mathrm{NaH}_{2} \mathrm{PO}_{4} \cdot 2 \mathrm{H}_{2} \mathrm{O}$ (all in analyticalgrade) were obtained from Sinopharm Chemical Reagent Co., Ltd. (Shanghai, China); $\mathrm{NaN}_{3}$ (in analytical-grade, used for anticorrosion) was provided by the Tianjin Fu Chen Chemical Reagent Factory. $\mathrm{D}_{2} \mathrm{O}$ (in analytical-grade) was produced from Cambridge Isotope Laboratories Inc., United States.

\section{Plasma Collection and Preparation}

A $1 \mathrm{~mL}$ venous blood sample was collected in a vacutainer tubes with ethylenediaminetetraacetic acid (EDTA) at 7 o' clock in the morning after $12 \mathrm{~h}$ of overnight fasting and then centrifuged at $10,000 \mathrm{rpm}$ for $10 \mathrm{~min}$ at $4^{\circ} \mathrm{C}$. The supernatant was stored at $-80^{\circ} \mathrm{C}$ until NMR analysis.

A total of $200 \mu \mathrm{L}$ plasma was mixed with $400 \mu \mathrm{L}$ of $\mathrm{K}_{2} \mathrm{HPO}_{4} / \mathrm{NaH}_{2} \mathrm{PO}_{4}$ buffer ( $45 \mathrm{mM}, \mathrm{pH} 7.4$ ) containing $50 \% \mathrm{D}_{2} \mathrm{O}$ and $0.9 \% \mathrm{NaCl}$ in a centrifuge tube $(1.5 \mathrm{~mL})$, after centrifugation $(12,000 \mathrm{rpm})$ for $10 \mathrm{~min}$, a total of $550 \mu \mathrm{L}$ of the supernatant was placed into a $5 \mathrm{~mm}$ NMR tubes directly for NMR analysis.

\section{NMR Analysis}

All ${ }^{1} \mathrm{H}$ NMR spectra were measured at $298 \mathrm{~K}$ on a Bruker AVIII 600 spectrometer (Bruker Biospin, Germany) equipped with Ultra cryogenic probe operating at $600.13 \mathrm{MHz}$ for ${ }^{1} \mathrm{H}$. 
A standard one-dimensional (1D) ${ }^{1} \mathrm{H}-\mathrm{NMR}$ spectra were recorded using a NOESYPR1D pulse sequence [recycle delay (RD) $-90^{\circ}-t_{1}-90^{\circ}-t_{\mathrm{m}}-90^{\circ}$-acquire] (Nicholson et al., 1995) to obtain all observed metabolites signals, CPMG pulse sequence [RD-90 $-\left(\tau-180^{\circ}-\tau\right)_{n}$-acquire] (Meiboom and Gill, 1958) to obtain small molecule metabolites signals and DIFFUSIONEDIT [RD- $90^{\circ}-\mathrm{G}_{1}-\tau-180^{\circ}-\mathrm{G}_{2}-\tau-90^{\circ}-\triangle-90^{\circ}-\mathrm{G}_{3}-\tau-180^{\circ}-\mathrm{G}_{4}-\tau-90^{\circ}-\mathrm{Te}-90^{\circ}-$ acquire] to obtain macromolecules metabolites. The following parameters were set for NMR detection in the experiments: $90^{\circ}$ pulse length $\left(P_{1}\right)$ was $11.2 \mu \mathrm{s}, \mathrm{PLW}_{1}$ was $10,-10[\mathrm{~W},-\mathrm{dBW}]$, spectral width was $20 \mathrm{ppm}$, the sampling point was $32 \mathrm{k}$, the acquisition time was $1.64 \mathrm{~s}$. 64 scans, 8 dummy scans. In the NOESYPR1D experiments, $t_{1}$ was $4 \mu \mathrm{s}$, mixing time $\left(D_{8}\right)$ was $100 \mathrm{~ms}$, relaxation decay time $\left(\mathrm{D}_{1}\right)$ was $2 \mathrm{~s}$, In the CPMG experiments, total echo time: $80 \mathrm{~ms}$, Echo evolution time (d20): $350 \mu \mathrm{s}$, Echo cycle $\left(\mathrm{L}_{4}\right): 100$. In the DIFFUSIONEDIT experiments, gradient recovery $\left(\mathrm{D}_{16}\right): 150 \mu \mathrm{s}$, diffusion time $\left(\mathrm{D}_{20}\right): 0.2 \mathrm{~s}$; Gradient strength (GPZ6) 85\%, gradient pulse length $\left(\mathrm{P}_{30}\right): 1100 \mu \mathrm{s}$.

For resonance assignment, a range of two-dimensional (2D) NMR spectra were acquired including ${ }^{1} \mathrm{H}-{ }^{1} \mathrm{H}$ correlation spectroscopy (COSY), ${ }^{1} \mathrm{H}_{-}{ }^{1} \mathrm{H}$ total correlation spectroscopy (TOCSY) NMR spectra, ${ }^{1} \mathrm{H}-{ }^{1} \mathrm{H}$ J-Resolved Spectroscopy (JRES), ${ }^{1} \mathrm{H}_{-}{ }^{13} \mathrm{C}$ heteronuclear single quantum correlation (HSQC) and ${ }^{1} \mathrm{H}-{ }^{13} \mathrm{C}$ heteronuclear multiple bond correlation spectra (HMBC). Briefly, in COSY and TOCSY experiments, the sampling point of $160\left(F_{1}\right)$ and $2048\left(F_{2}\right)$, spectral width of $10.5 \mathrm{ppm}\left(F_{1}\right)$ and $10.5 \mathrm{ppm}\left(F_{2}\right), 90^{\circ}$ pulse length $\left(P_{1}\right)$ of $11.87 \mathrm{~s}$. TOCSY 2D NMR spectra were acquired with MLEV as the spin lock pulse and the spin-lock time of $80 \mathrm{~ms} .{ }^{1} \mathrm{H}-{ }^{1} \mathrm{H}$ JRES were acquired with the sampling point of $64\left(F_{1}\right)$ and 2048 $\left(F_{2}\right)$ the spectral widths were $0.1 \mathrm{ppm}$ in the $F_{1}$ dimension and $10.5 \mathrm{ppm}$ in the $F_{2}$ dimension. ${ }^{1} \mathrm{H}-{ }^{13} \mathrm{C}$ HSQC $2 \mathrm{D}$ NMR spectra were acquired using composite pulse broad band decoupling (globally alternating optimized rectangular pulses, GARP), the sampling points were $128\left(F_{1}\right)$ and $2048\left(F_{2}\right)$, with the spectral widths of $220 \mathrm{ppm}\left(F_{1}\right)$ and $10.5 \mathrm{ppm}\left(F_{2}\right) .{ }^{1} \mathrm{H}_{-}{ }^{13} \mathrm{C}$ HMBC $2 \mathrm{D}$ NMR spectra were acquired into 128 data points $\left(F_{1}\right)$ and 2048 data points $\left(F_{2}\right)$, the spectral widths were $220 \mathrm{ppm}\left(F_{1}\right)$ and $10.5 \mathrm{ppm}\left(F_{2}\right)$.

\section{Data Pre-processing of NMR Spectra and Multivariate Data Analysis}

Baseline and phase corrections for the NMR spectra were manually achieved using mestrenova (version 9.0.1, Mestrelab Research, Santiago de Compostela, Spain). The peak of L-lactate with a chemical shift at $\delta 1.33$ was used as a spectral reference for plasma. The spectral region of $\delta 0.5-9.5$ was segmented into $0.002 \mathrm{ppm}$ (He et al., 2009) chemical shift buckets, the region at $\delta 4.70-5.00$ and $\delta 5.6-6.0$ were discarded to eliminate the effects of imperfect water suppression and urea signal, respectively. The regions of EDTA-Ca resonance ( $\delta 2.55-2.58$, \$3.07-3.17), EDTA-Mg resonance ( $82.68-2.70,83.23-3.26)$, FreeEDTA resonance $(\delta 3.6-3.63)$ were removed. The integral value of each spectrum was used as input variables for the subsequent statistical analysis.
Principal component analysis (PCA) and partial least squares discrimination analysis (PLS-DA) were performed to examine intrinsic variation of the NMR spectral data using SIMCAP 14.0 (Umetrics, Sweden). Each point on the scores plot is defined by the spectrum of an individual sample. Then, RFs in software MATLAB (2010b, The MathWorks Inc., Natick, MA, United States) were applied to uncover the underlying structure of this data, multidimensional scaling (MDS) was employed to map the proximity into a lower-dimensional space.

A one-way analyses of variance (ANOVA) with a Bonferroni correction of the SPSS 17.0 for Windows (SPSS Inc., Chicago, IL, United States) was used for significance analysis. $P$-values less than 0.05 were considered significant.

\section{Random Forest}

There are two powerful machine learning techniques advantages with RF: bagging and random feature selection. In bagging, each tree is trained on a bootstrap sample of the training data, and predictions are made by majority vote of trees. Instead of using all features, RF randomly selects a subset of features to split at each node when growing a tree. To assess the prediction performance of the RF algorithm, it performs a type of cross-validation in parallel with the training step by using the so-called out-of-bag (OOB) samples. On average, each tree is grown using about $2 / 3$ of the training data, leaving about $1 / 3$ as OOB. The RF algorithm can be stated as follows (Huang et al., 2013):

Draw $n_{\text {tree }}$ bootstrap samples from the original data, $n_{\text {tree }}$ is the number of ensemble tree in RF. The $n_{\text {tree }}$ is equal to 2000 in this study.

For all bootstrap samples, grow an un-pruned classification or regression tree, with the following modification: at each node, rather than choosing the best split among all variables, randomly sample mtry of the variables and choose the best split from among those variables (bagging can be thought of as the special case of RFs obtained when mtry $=p$, the number of variables). In general, mtry is simply a number (positive integer) between 1 and $p$.

Predict new data by aggregating the predictions of $n_{\text {tree }}$ (i.e., majority votes for classification).

\section{Variable Importance}

Random forest algorithm has the ability to estimate feature importance. A measure of how each feature contributes to the prediction performance of RF algorithm can be calculated in the course of the training. The important scores can be used to identify biomarkers. The frequently used type of RF algorithm to measure feature importance is the mean decrease in classification based on permutation. The prediction accuracy after permutation is subtracted from the prediction accuracy before permutation and averaged over all trees in the forest to give the permutation importance value. In the current research, the mean decrease in classification accuracy was accepted to measure variable importance.

\section{Proximity Measure}

Proximity matrix is the important feature of RF algorithm which can be used to identify structure in the data. RF algorithm not only generates variable-related information such as variable 
importance measures, but also calculates the proximities between samples. All samples in the original data set are classified by the forest. The proximity between two samples is calculated as the number of times the two samples end up in the same terminal node of a tree, divided by the number of trees in the forest. The resulting matrix is symmetric with diagonal element equal to 1 and off-diagonal elements ranging from 0 to 1 . The proximities between similar samples are always high. Proximity scores may also be used to construct MDS plots. MDS plots aim to visualize the similarity or dissimilarity (calculated as 1 proximity) between samples.

\section{RESULTS}

\section{Demographic and Clinical Characteristics of the Samples}

Demographic and clinical data of the subjects were summarized in Table 1. There was no significant difference in age, gender ratio, body mass index and other clinical feature between the three groups $(P>0.05)$, as assessed by Friedman's ANOVA.

\section{${ }^{1} \mathbf{H}$ NMR Spectrum of Plasma Samples}

A typical $1 \mathrm{D}{ }^{1} \mathrm{H}$ NMR CMPG spectrum of plasma samples from (A) control and (B) CHD group were shown in Figure 1. Resonance assignments of metabolites were made according to the literature and confirmed by 2D NMR spectra. 69 metabolites were identified, covering amino acids, organic acids, lipids, glucose, salts, choline and urea. In addition, Tricarboxylic acid (TCA) cycle metabolites, such as succinate and citrate were included. Visual inspection of the $1 \mathrm{D}{ }^{1} \mathrm{H}$ NMR CMPG spectra showed clear differences in overall composition between CHD patients and control (Figure 1). However, these qualitative observations were all by visual inspection, multivariate data analysis of NMR spectra were performed to recover the characteristics of metabolic patterns of CHD (included BSS and PS) and control.

\section{Classification Using PCA, PLS-DA, and RF}

Principal component analysis on plasma CPMG spectra were applied to observe the classification of $\mathrm{CHD}$ patients and

TABLE 1 | Demographic and clinical characteristics of the samples.

\begin{tabular}{lcccc}
\hline & $\begin{array}{c}\text { HC } \\
(\boldsymbol{n}=\mathbf{2 6})\end{array}$ & $\begin{array}{c}\text { CHD-BSS } \\
(\boldsymbol{n}=\mathbf{2 7})\end{array}$ & $\begin{array}{c}\text { CHD-PS } \\
(\boldsymbol{n}=\mathbf{3 1})\end{array}$ & $\boldsymbol{P}$-value \\
\hline Age, mean $\pm \mathrm{SD}$, years & $53.73 \pm 11.39$ & $53.70 \pm 13.20$ & $54.97 \pm 10.88$ & 0.896 \\
Male, $n(\%)$ & $20(76.92)$ & $19(70.37)$ & $18(58.06)$ & 0.298 \\
Body mass index, & $24.42 \pm 2.57$ & $25.59 \pm 3.89$ & $23.61 \pm 2.58$ & 0.054 \\
mean $\pm \mathrm{SD}\left(\mathrm{kg} / \mathrm{m}^{2}\right)$ & & & & \\
Active smoking, $n(\%)$ & $10(38.46)$ & $11(40.74)$ & $10(32.26)$ & 0.785 \\
Diabetes mellitus, $n(\%)$ & $5(19.23)$ & $9(33.33)$ & $10(32.26)$ & 0.445 \\
Hypertension, $n(\%)$ & $8(30.77)$ & $9(33.33)$ & $11(35.48)$ & 0.932 \\
Dyslipidemia, $n(\%)$ & $7(26.92)$ & $14(51.85)$ & $16(51.61)$ & 0.106
\end{tabular}

controls. The first two principal components were plotted to present the distribution of the three groups (Figure 2).

As visually observed, the three groups were totally overlapped in the PCA scores plot, suggesting that the unsupervised PCA method could not extract useful information in the NMR CMPG data. PLS-DA was also used to show the classification of the three groups, as presented in Figure 2, and only the differences between the CHD and control groups could be observed. As for BSS and PS of CHD patients, they were completely overlapped, and had no separation trend. PCA and PLS-DA on plasma NOESY and BPP-LED spectra, and model verification of PLS-DA on plasma CMPG spectra, BPP-LED spectra and NOESY spectra were shown in Supplemetary Figures S1-S5.

Then, RF method was used to explore the underlying characteristics of this NMR data. 2000 trees were grown, during the trees growing, proximities were computed for the cases. Similar cases may fall into the same terminal node or derive from the same parent. In order to better present the differences of the samples, MDS was employed to map the proximity into a lower dimensional space. OOB estimate of error rate and fivefold cross validation were used to evaluate the stability of the forest tree model. It could be seen from Figure 3, the OOB error rate did not decrease with the number of trees constructed, the RF algorithm could avoid overfitting to a certain extent, and the area under the curve of ROC was 0.96. As shown in Figure 3A, the controls were far away from other two groups. Furthermore, an obvious distinction between CHD-BSS and CHD-PS was observed in the MDS plot. Figure 3B showed the OOB error rate.

It can be seen from Figure 3, the OOB error rate did not decrease with the number of trees constructed, the RF algorithm could avoid overfitting to a certain extent.

\section{Discovery of Plasma ${ }^{1} \mathrm{H}-\mathrm{NMR}$ Potential Biomarkers in CHD Patients and CHD-BSS Patients}

Metabolic biomarker discovery is an important aim of metabolomics studies. In model construction, the purpose of variable selection is to find the best combination of variables, which provide the best classification result (Huang et al., 2013). A measure of how each feature contributes to the prediction performance of RF can be calculated in the course of training, and its importance score (VIM) was obtained. VIM was used to measure the contribution of the variable to the classification. Figure 4 showed MDS plots and the variable importance. The feature importance was set to 0.1 , from the bar plot of variable importance, we found that some metabolites make a contribution to the classification of controls and CHD samples (Figure 4a), the peak area quantitative of each spectrum were manually achieved by segmenting into $0.002 \mathrm{ppm}$ in mestrenova, then $t$-test was implemented to test the significant of these metabolites and the result was summarized in Table $2(P<0.05)$. Finally, we obtained 12 characteristic metabolites, including lysine (1.40 ppm), glutamine (2.45 ppm), taurine (3.28 ppm), tyrosine (3.93 ppm), phenylalanine (3.96 ppm), histidine (4.0 ppm), lipid (2.0 ppm), citrate (2.55 ppm), choline (3.20 ppm, $4.05 \mathrm{ppm})$, lactate $(4.11 \mathrm{ppm}), \alpha$-Glucose $(5.23 \mathrm{ppm}, 3.39 \mathrm{ppm}, 3.56 \mathrm{ppm}$, 


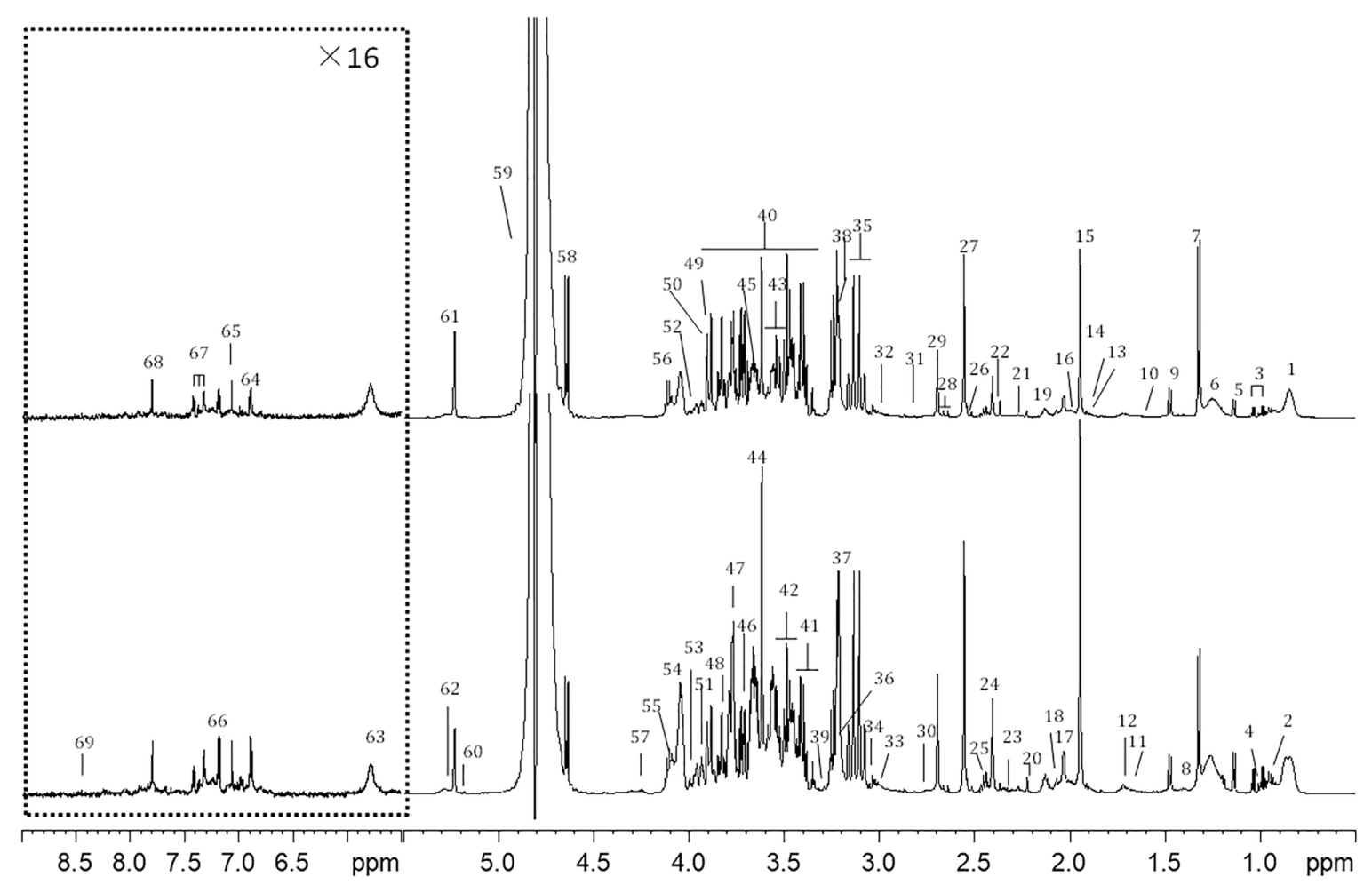

FIGURE 1 | Typical cpmgpr1d spectra (600 MHz) of (A) CHD patient, (B) control from plasma samples (the region at $85.5-9.0$ was expanded for 16 times). Key: 1 , lipid $\left(\mathrm{CH}_{3}\right) ;$, leucine and isoleucine; 3, valine; 4. isoleucine; 5, 3-hydroxybutyrate; 6, lipid $\left(\mathrm{CH}_{2}\right) ; 7$, lactate; 8, lysine; 9, alanine; 10, lipid $\left(\mathrm{CH}_{2} \mathrm{CH}_{2} \mathrm{CO}\right) ; 11$, lysine and arginine; 12, leucine; 13, lysine and arginine; 14, acetate; 15, acetic acid $\left(\mathrm{CH}_{3} \mathrm{COOH}\right) ; 16$, lipid $\left(\mathrm{CH}_{2} \mathrm{C}=\mathrm{C}\right) ; 17, \mathrm{~N}$-acetyl glycoproteins (NAG) 18, glutamate; 19, glutamine; 20, acetoacetate; 21, lipid $\left(\mathrm{CH}_{2} \mathrm{C}=\mathrm{O}\right)$; 22, pyruvate; 23, glutamate; 24, succinate; 25, glutamine; 26, citrate; 27, Ca-EDTA; 28, citrate; 29, Mg-EDTA; 30, lipid $\left(\mathrm{C}=\mathrm{CCH}_{2} \mathrm{C}=\mathrm{C}\right)$; 31, trimethylamine; 32, lysine; 33, creatine; 34, creatinine; 35, Ca-EDTA; 36, choline and GPC, glycerophosphocholine (GPC) and phosphocholine (PC); 37, free-EDTA; 38, Mg-EDTA; 39, taurine; 40, glucose and amino acid; 41, $\alpha$-glucose and taurine; 42, $\beta$-glucose; 43, $\alpha$-glucose; 44, free-EDTA; 45, choline; 46, $\alpha$-glucose; 47, $\beta$-glucose; 48, $\alpha$-glucose; 49, $\alpha$-glucose; $50, \beta$-glucose; 51 , tyrosine; 52 , phenylalanine; 53 , histidine; 54 , choline; 55 , TG; 56, lactate; 57, TG; 58, $\beta$-glucose; 59, $\mathrm{H}_{2} \mathrm{O} ; 60, \mathrm{TG} ; 61, \alpha$-glucose; 62 , lipid $(\mathrm{CH}=\mathrm{CH}) ; 63$, urea; 64 , tyrosine; 65 , histidine; 66 , tyrosine; 67 , phenylalanine; 68 , histidine; 69, formate.

$3.71 \mathrm{ppm}, 3.83 \mathrm{ppm}, 3.89 \mathrm{ppm})$, and $\beta$-Glucose (3.47 ppm, $3.72 \mathrm{ppm})$ related to the CHD patients. These metabolites involved in the key metabolic pathways are shown in Figure 5.

The variable importance measures of samples obtained by RF models revealed that in the plasma of CHD patients contained significantly higher levels of some amino acids, including lysine, glutamine, taurine, tyrosine, phenylalanine and histidine compared to the controls. Plasma lipid, lactate, $\alpha$-glucose and $\beta$-glucose in CHD patients were higher when compared with controls $(P<0.01)$. However, citrate and choline of plasma from CHD patients were lower than those from angiography normal (controls) $(P<0.01)$.

In order to explore potential metabolite biomarkers of CHDBSS patients, RF methodology was applied to extract important variables of the control group and CHD group. The feature importance was enhanced to 0.2 to obtain the most important variables. The variable importance measures of each two groups obtained by RF models were shown in Figure 4. The important variables $(P<0.01)$ of the each two groups were shown in Table 3, we found that $72 /$ choline, $73 / \beta$-glucose, $74 / \alpha$-glucose, $75 / \alpha$-glucose, $77 / \alpha$-glucose, $78 /$ tyrosine could be the potential metabolite biomarkers of CHD-BSS patients.

\section{DISCUSSION}

The use of the analytical techniques, ${ }^{1} \mathrm{H}-\mathrm{NMR}$, was feasible to study the metabonomic differences between angiography normal and CHD patients. ${ }^{1} \mathrm{H}-\mathrm{NMR}$ analysis enabled the identification of a total of 12 metabolites as contributors to the discrimination of controls and CHD patients. We found that the potential biomarkers were principally correlated to lipid metabolism dysfunction, energy metabolism dysfunction, amino acid dysfunction and glucose metabolism dysfunction in the pathological development of CHD. In our previous studies using LC-MS, we found 27 potential biomarkers which were principally involved in arachidonic acid metabolism, amino acid metabolism, purine metabolism, pyrimidine metabolism, steroid biosynthesis and linoleic acid metabolism that discriminated CHD patients from healthy controls. In those potential biomarkers, citric acid and phenylalanine were found in the current research with NMR technology. Integration of several metabonomics technologies could provide more comprehensive biomarker information.

Choline is an important methyl donor, precursor of acetylcholine, and it is needed for lipid metabolism (Ueland, 2011). 

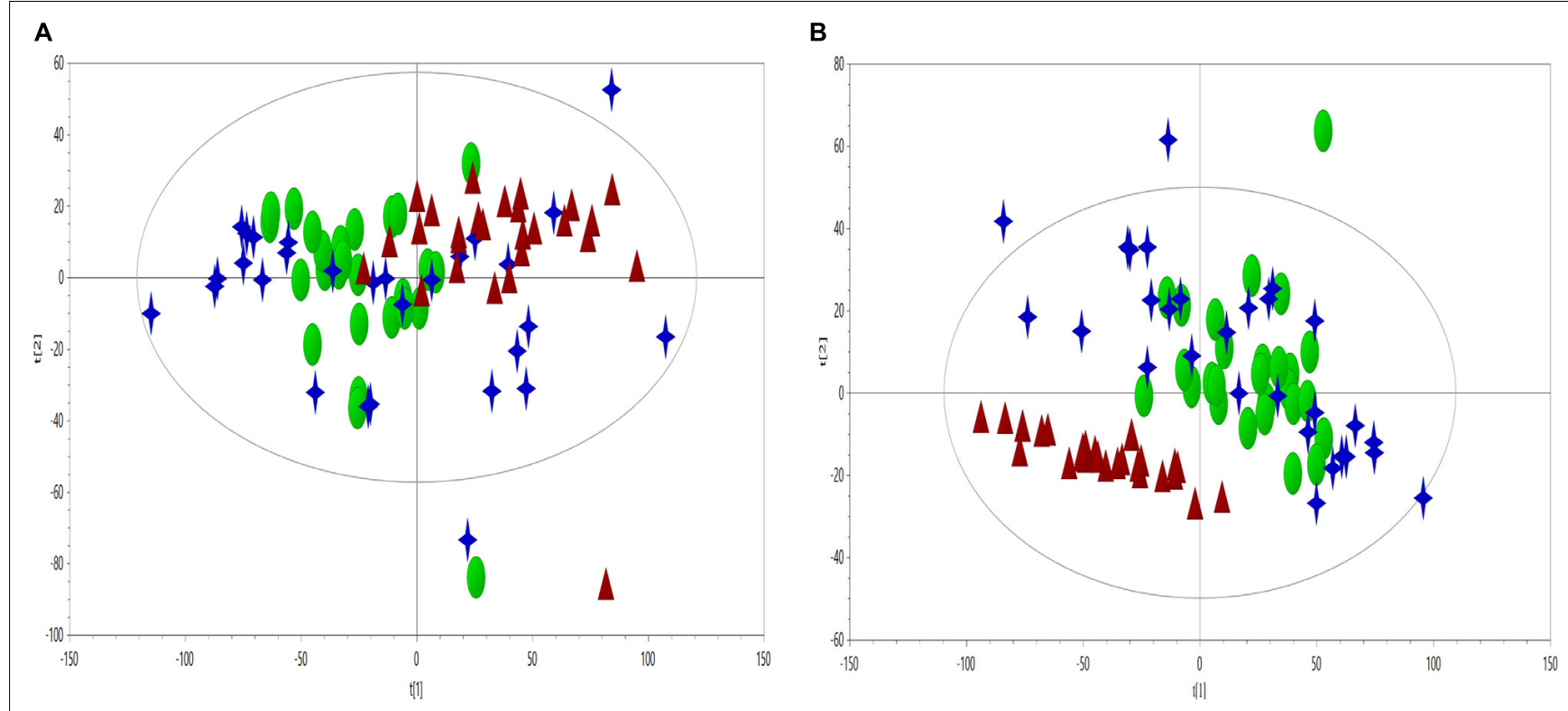

FIGURE 2 | PCA scores plot $\left(\mathbf{A}, R^{2} X=0.636, Q^{2}=0.560\right)$ and PLS-DA scores plot $\left(\mathbf{B}, R^{2} X=0.599, Q^{2}=0.318, R^{2} Y=0.37\right)$ derived from NMR data to compare the metabolome of the control (triangles, red), CHD-BSS (circles, green) and CHD-PS (stars, blue).
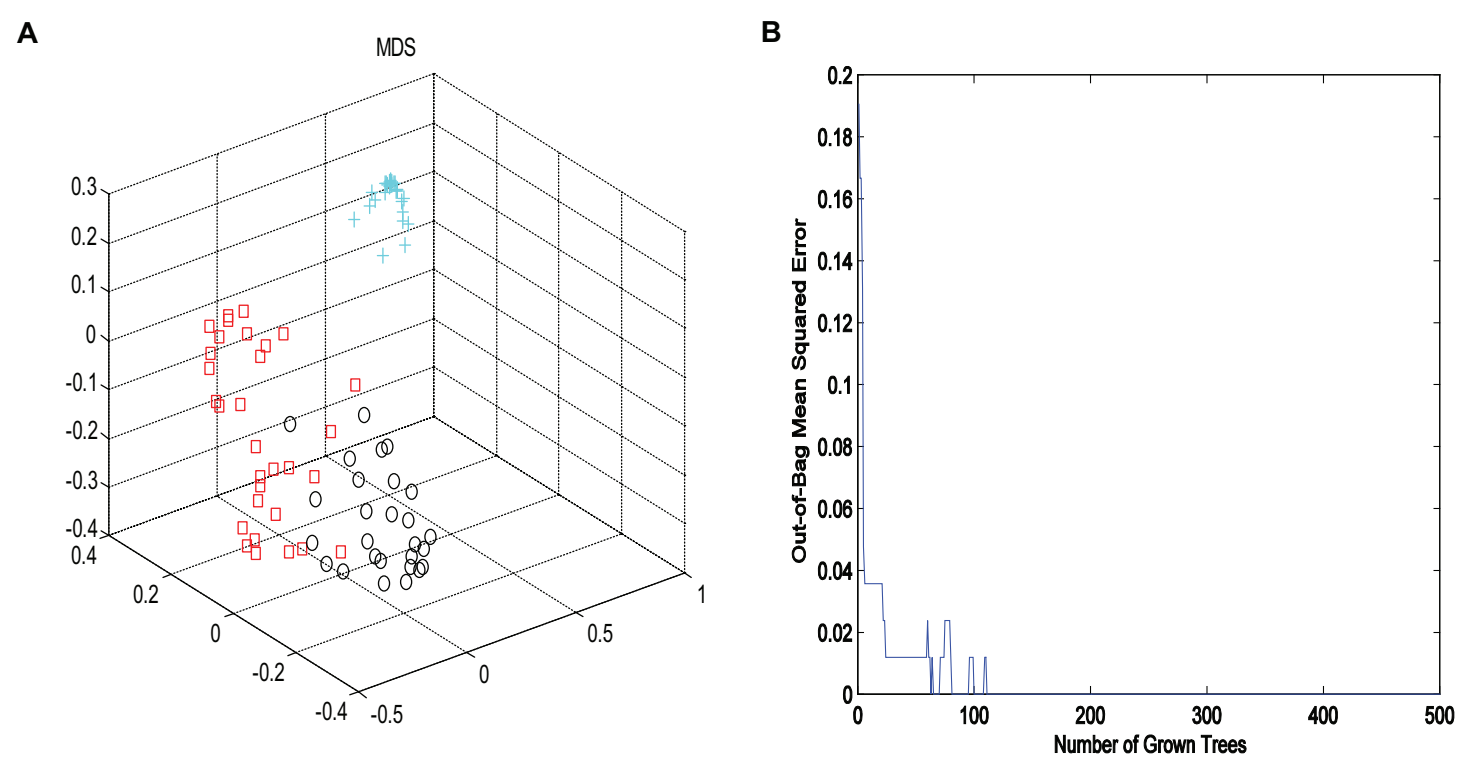

FIGURE 3 | The MDS plot (A) for plasma profiles derived from NMR data for control (cross, blue), CHD-BSS (circles, black) and CHD-PS (squares, red). Plot of OOB error for RF classification of the three groups (B).

Choline can be converted to betaine in a two-step enzymatic reaction occurring mainly in the mitochondria of liver cells. Choline or betaine may be important for lowering plasma homocysteine concentrations (Steenge et al., 2003), which is associated with increased risk of cardiovascular disease (CVD) (Homocysteine Studies Collaboration, 2002). LC-HRMS method was applied to measure the plasma concentrations of choline in a cohort of 339 patients undergoing coronary angiography for the evaluation of suspected coronary artery disease, the results show plasma levels of choline are significantly lower in patients with a history of acute myocardial infarction as compared to those without such history (Mueller et al., 2015). Brindle et al. (2002) have shown that choline contributed most strongly to the discrimination of the CHD and control group. Choline (Oliver and Martin, 2010) are emerging biomarkers in acute coronary syndrome. Our study 

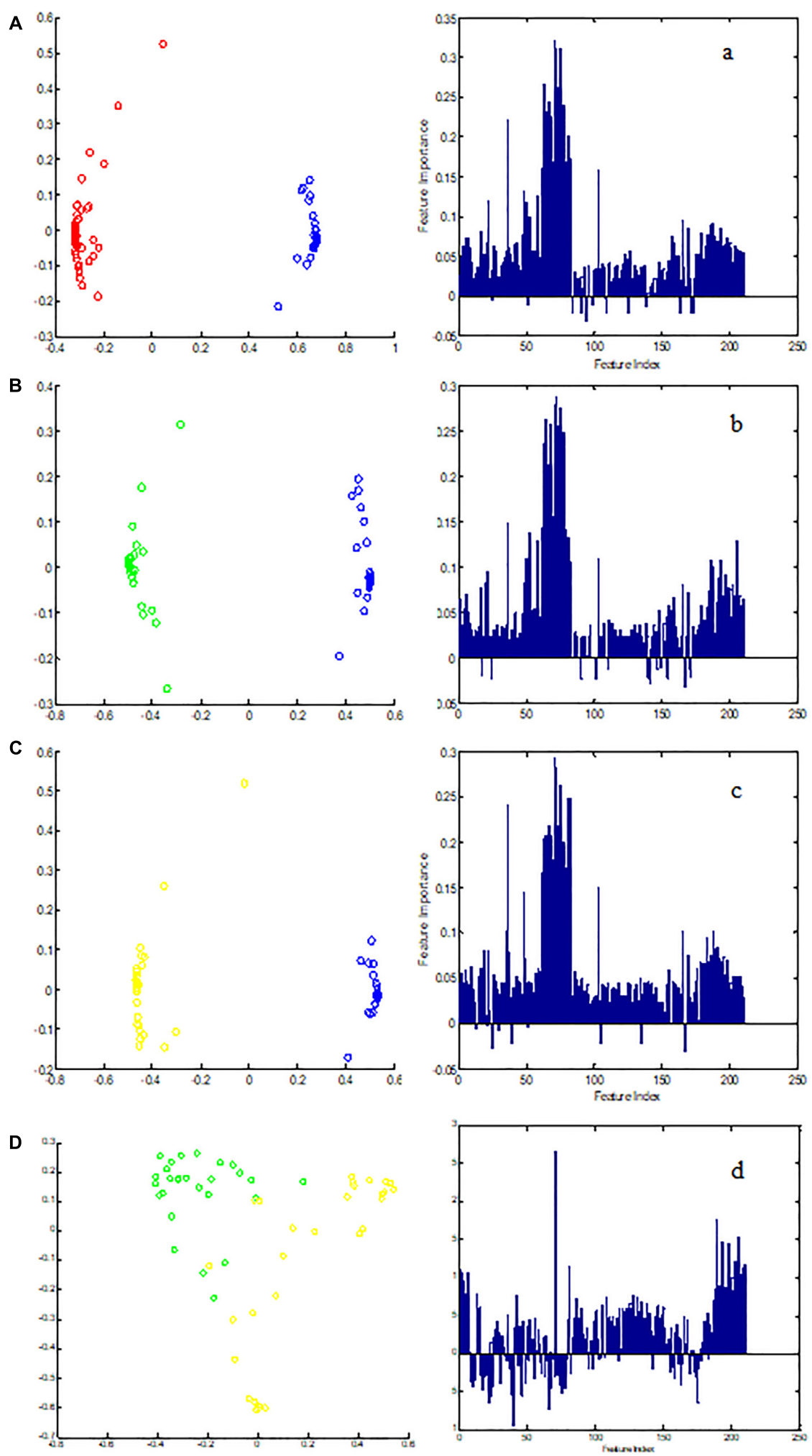

FIGURE 4 | The MDS plot of (A) control (blue) and CHD (red), (B) control and CHD-BSS (green), (C) control and CHD-NBSS (yellow), and (D) CHD-BSS and CHD-NBSS. The VIM plot of (a) control and CHD, (b) control and CHD-BSS, (c) control and CHD-NBSS, and (d) CHD-BSS and CHD-NBSS obtained by random forest were shown in the right. 
TABLE 2 | The summaries of the metabolites that contributed to the clustering of CHD patients and angiography normal (controls) $(P<0.01)$.

\begin{tabular}{|c|c|c|c|c|c|}
\hline $\begin{array}{l}\text { Important } \\
\text { variables }\end{array}$ & $\begin{array}{l}\text { Chemical } \\
\text { shift (ppm) }\end{array}$ & $\begin{array}{l}\text { Potential } \\
\text { biomarker }\end{array}$ & $\begin{array}{c}\text { Peak area } \\
\text { in controls }\end{array}$ & $\begin{array}{l}\text { Peak area in } \\
\text { CHD patients }\end{array}$ & $P$ \\
\hline 22 & 1.403 & Lysine & $12.31 \pm 9.82$ & $39.60 \pm 35.78$ & 0.000 \\
\hline 48 & 2.443 & Glutamine & $69.39 \pm 10.88$ & $164.45 \pm 99.36$ & 0.000 \\
\hline 50 & 2.523 & Citrate & $55.25 \pm 10.79$ & $35.83 \pm 11.83$ & 0.000 \\
\hline 62 & 3.203 & Choline & $337.94 \pm 55.40$ & $208.86 \pm 72.96$ & 0.000 \\
\hline 64 & 3.323 & $\alpha$-glucose & $8.26 \pm 7.97$ & $45.48 \pm 19.54$ & 0.000 \\
\hline 66 & 3.403 & $\alpha$-glucose & $85.09 \pm 19.24$ & $236.05 \pm 126.66$ & 0.000 \\
\hline 67 & 3.443 & $\beta$-glucose & $111.78 \pm 19.55$ & $387.09 \pm 201.84$ & 0.000 \\
\hline 68 & 3.483 & $\beta$-glucose & $134.09 \pm 28.85$ & $513.31 \pm 292.54$ & 0.000 \\
\hline 69 & 3.523 & $\alpha$-glucose & $132.77 \pm 26.66$ & $368.55 \pm 210.79$ & 0.000 \\
\hline 70 & 3.563 & $\alpha$-glucose & $180.07 \pm 30.66$ & $572.60 \pm 332.92$ & 0.000 \\
\hline 74 & 3.763 & $\alpha$-glucose & $79.92 \pm 17.81$ & $260.75 \pm 112.37$ & 0.000 \\
\hline 75 & 3.803 & $\alpha$-glucose & $73.26 \pm 14.69$ & $652.03 \pm 417.20$ & 0.000 \\
\hline 76 & 3.843 & $\alpha$-glucose & $89.50 \pm 20.28$ & $241.12 \pm 117.13$ & 0.000 \\
\hline 77 & 3.883 & $\alpha$-glucose & $77.35 \pm 14.27$ & $197.34 \pm 247.43$ & 0.016 \\
\hline 78 & 3.923 & Tyrosine & $107.86 \pm 18.32$ & $274.94 \pm 117.29$ & 0.000 \\
\hline 79 & 3.963 & phenylalanine & $44.95 \pm 14.34$ & $143.55 \pm 82.69$ & 0.000 \\
\hline 80 & 4.003 & histidine & $54.72 \pm 14.74$ & $106.16 \pm 44.69$ & 0.000 \\
\hline 81 & 4.043 & Choline & $34.25 \pm 15.40$ & $253.45 \pm 174.81$ & 0.000 \\
\hline 82 & 4.083 & Lactate & $58.42 \pm 17.47$ & $395.31 \pm 295.24$ & 0.000 \\
\hline 103 & 5.243 & $\alpha$-glucose & $-33.66 \pm 29.03$ & $58.58 \pm 90.43$ & 0.000 \\
\hline
\end{tabular}

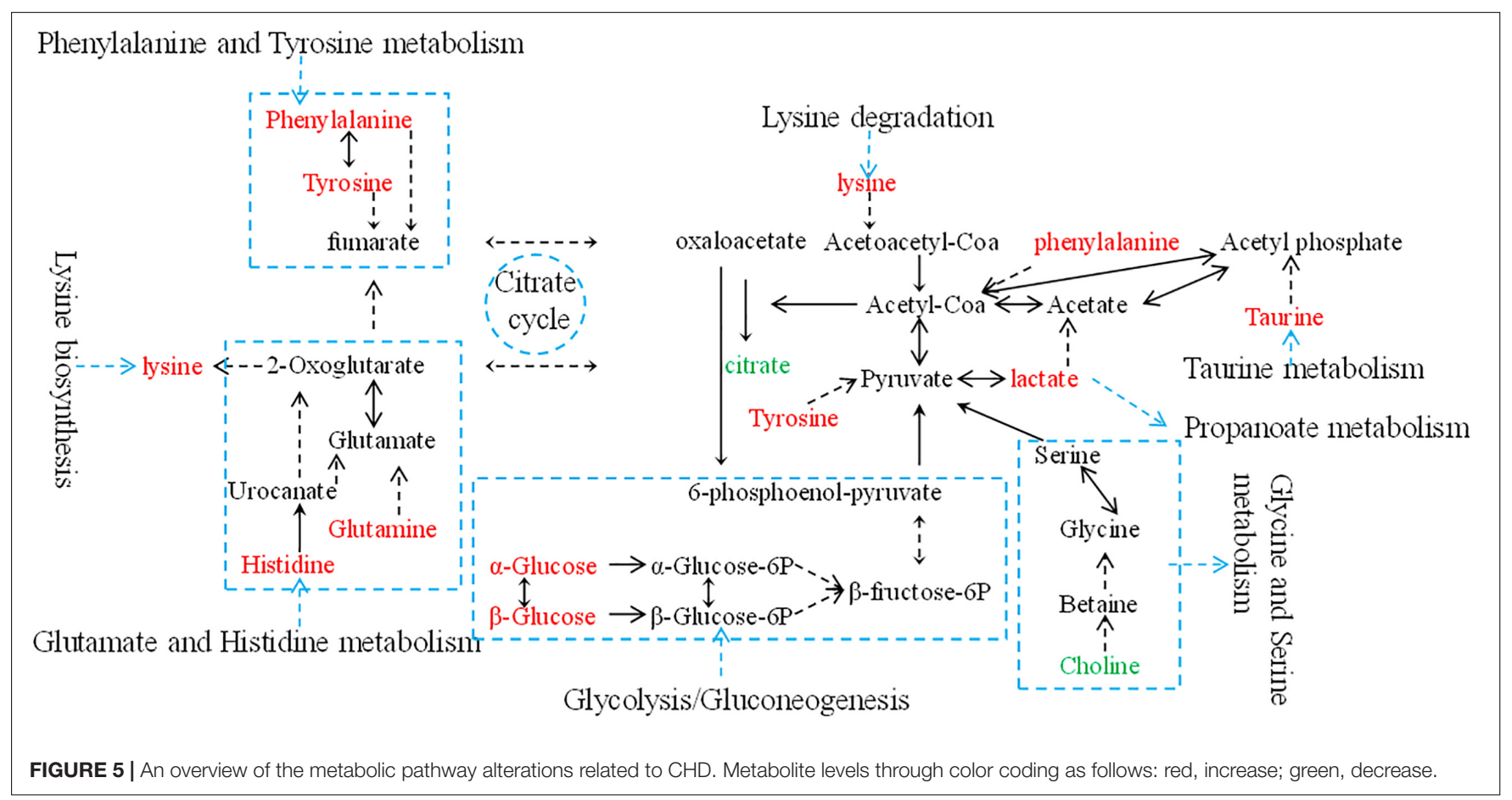


TABLE 3 | The important variables $(P<0.01)$ of the each two groups.

\begin{tabular}{ll}
\hline Group & \multicolumn{1}{c}{ Important variables/metabolites } \\
\hline Control-CHD & 36/lipid, 63/taurine, $64 / \alpha$-glucose, $67 / \beta$-glucose, $68 / \beta$-glucose, \\
& $71 /$ choline, $72 /$ choline, $73 / \beta$-glucose, $74 / \alpha$-glucose, \\
& $75 / \alpha$-glucose, $77 / \alpha$-glucose, $78 /$ tyrosine, $81 /$ choline \\
Control-BSS & 63/taurine, 64/ $\alpha$-glucose, $67 / \beta$-glucose, $68 / \beta$-glucose, \\
& $71 /$ choline, $72 /$ choline, $73 / \beta$-glucose, $74 / \alpha$-glucose, \\
& $75 / \alpha$-glucose, $77 / \alpha$-glucose, $78 /$ tyrosine \\
Control-PS & 36/lipid, 64/ $\alpha$-glucose, 67/ $\beta$-glucose, 68/ $\beta$-glucose, $71 /$ choline, \\
& $72 /$ choline, $73 / \beta$-glucose, $75 / \alpha$-glucose, $81 /$ choline, $82 /$ lactate \\
BSS-PS & $71 /$ choline \\
\hline
\end{tabular}

demonstrated that plasma choline levels in patients with $\mathrm{CHD}$ remarkably decreased, suggesting the relationship between choline and CHD risk.

Abnormal metabolism of the lactate and citrate were the indications of energy metabolism abnormality. Lactate, which is an end product of glucose anaerobic glycolysis, is reportedly a useful indicator for ischemia according to the clinical and scientific studies. It has been found that lactate was remarkably increased in plasma of patients with CHD in this study which suggested that glycolysis was activated by myocardial ischemia and hypoxia. Previous studies (Barderas et al., 2011; Rowland et al., 2013) have shown an obvious lactate increased in CHD patients. The citric acid cycle plays a central role in oxidative phosphorylation in the myocardium. In the setting of acute ischemia, preservation of citric acid cycle intermediates becomes of paramount importance to defend ATP production. Sabatine et al. (2005) have demonstrated highly statistically significant changes in circulating levels of metabolites belonging to the citric acid pathway, including citrate. In our study, the citrate level was also found lower in the CHD group than in the control group.

In the metabolic processes of the amino acids, plasma lysine, glutamine, taurine, tyrosine, phenylalanine and histidine of the CHD patients had increased significantly in this study, especially taurine and tyrosine. It has been found in several metabolic studies that amino acid metabolism is frequently abnormal in patients with CHD. The surplus amino acids provided adequate raw materials for the synthesis of lipid, thus abnormal lipid metabolism of CHD patients had been aggravated at the same time.

Our study found that the level of $\alpha$-glucose, $\beta$-glucose in the plasma of $\mathrm{CHD}$ patients was higher than that in the control group. Glucose, as a short-term marker for glycemic control (Dungan, 2008), could indicate that significant disorders of glucose metabolism were happened in CHD patients, and CHD individuals at higher risk for developing diabetes or insulin resistance.

Furthermore, this study showed a clear metabonomic difference between CHD-BSS group and CHD-PS group (Figure 4), indicating CHD-BSS and CHD-PS were different metabolism patterns. Choline, $\beta$-glucose, $\alpha$-glucose and tyrosine were considered as potential biomarkers of CHD-BSS in this study. Choline can prevent CVD by preventing the deposition of TC in the inner wall of blood vessels and improving the absorption and utilization of fat. A proteome study (Zhao et al., 2008) found that there was lipid metabolism disorder in patients with CHD-BSS. Apolipoproteins in plasma of patients with CHD-BSS decreased. Apolipoproteins A IV is involved in the reverse transport of cholesterol. In our previous studies, amino acids, fatty acids, purine and so on were selected as a panel of candidate biomarkers of CHD-BSS based on GC-MS (Wei et al., 2013) and LC-MS (Zhao et al., 2014) technology, integration of several technologies could provide more comprehensive classification and biomarker information. It suggested that the potential biomarkers revealed by the three techniques were supplementary. In addition, PCA and PLS-DA method could not extract useful information in the NMR CMPG data for patternrecognition analyses of biological samples. The RF method could be used to explore more the underlying characteristics of biological samples in this study. Therefore, it is important to develop more efficient pattern recognition approach for the analysis of complex metabolomics data. CHD-BSS is not exist alone in the clinics, so one limitation is that CHD-BSS group included in the study is also mixed with other syndromes, such as qi deficiency, yin deficiency, etc., we will enroll patients of BSS with different diseases and adopt targeted LC-MS/MS analysis to verify the potential markers of BSS in future studies.

\section{CONCLUSION}

Our study demonstrated a clear metabonomic difference between CHD-BSS group and CHD-PS group, indicating CHD-BSS and CHD-PS were different metabolism patterns. Choline, $\beta$-glucose, $\alpha$-glucose and tyrosine were considered as potential biomarkers of CHD-BSS. It is also worth noting that RF and MDS could be used to explore more the underlying characteristics of biological samples in this study, and RF could effectively mine the pattern information hidden in the complex metabolomics data.

\section{DATA AVAILABILITY}

All datasets generated for this study are included in the manuscript and the Supplementary Files.

\section{ETHICS STATEMENT}

This study was carried out in accordance with the recommendations of "The IRB of Xiangya Hospital, Central South University" with written informed consent from all subjects. All subjects gave written informed consent in accordance with the Declaration of Helsinki. The protocol was approved by the "The IRB of Xiangya Hospital, Central South University."

\section{AUTHOR CONTRIBUTIONS}

D-SW conceived and designed the research. L-LZ and W-BW performed the following research projects. X-JQ, R-ML, and D-SW contributed toward the identification of disease and 
ZHENG types. L-LZ conducted data pre-processing of the NMR spectra, data analysis, and drafted the manuscript. W-BW and D-SW contributed toward the results validation. All authors revised the manuscript.

\section{FUNDING}

This research was supported by the National Natural Science Foundation of China, China (No. 81173198, 81373553, and 81703963).

\section{REFERENCES}

Ansar, S., Koska, J., and Reaven, P. D. (2011). Postprandial hyperlipidemia, endothelial dysfunction and cardiovascular risk: focus on incretins. Cardiovasc. Diabetol. 10:61. doi: 10.1186/1475-2840-10-61

Barderas, M. G., Laborde, C. M., Posada, M., Cuesta, F., Zubiri, I., Vivanco, F., et al. (2011). Metabolomic profiling for identification of novel potential biomarkers in cardiovascular diseases. J. Biomed Biotechnol. 2011:790132. doi: 10.1155/ 2011/790132

Breiman, L. (2001). Random forests. Mach. Learn. 45, 5-21.

Brindle, J. T., Antti, H., Holmes, E., Tranter, G., Nicholson, J. K., Bethell, H. W., et al. (2002). Rapid and noninvasive diagnosis of the presence and severity of coronary heart disease using $1 \mathrm{H}-\mathrm{NMR}$-based metabonomics. Nat. Med. 8, 1439-1444.

Dungan, K. M. (2008). 1,5-anhydroglucitol (GlycoMark) as a marker of shortterm glycemic control and glycemic excursions. Expert Rev. Mol. Diagn. 8, 9-19. doi: 10.1586/14737159.8.1.9

He, Q., Kong, X., Wu, G., Ren, P., Tang, H., Hao, F., et al.(2009). Metabolomic analysis of the response of growing pigs to dietary L-arginine supplementation. Amino Acids 37, 199-208. doi: 10.1007/s00726-008-0192-9

Homocysteine Studies Collaboration (2002). Homocysteine and risk of ischemic heart disease and stroke: a meta-analysis. JAMA 288:2015. doi: 10.1001/jama. 288.16.2015

Huang, J. H., Xie, H. L., Yan, J., Cao, D. S., Lu, H. M., Xu, Q. S., et al. (2013). Interpretation of type 2 diabetes mellitus relevant GC-MS metabolomics fingerprints by using random forests. Anal. Methods 5, 4883-4889. doi: 10. $1039 / \mathrm{c} 3 a y 40379 \mathrm{c}$

Lacoste, L., Lam, J. Y., Hung, J., Letchacovski, G., Solymoss, C. B., and Waters, D. (1995). Hyperlipidemia and coronary disease correction of the increased thrombogenic potential with cholesterol reduction. Circulation 92, 3172-3177. doi: 10.1161/01.cir.92.11.3172

Lindon, J. C., Holmes, E., Bollard, M. E., and Stanley, E. G. (2004). Metabonomics technologies and their applications in physiological monitoring, drug safety assessment and disease diagnosis. Biomarkers 9, 1-31. doi: 10.1080/ 13547500410001668379

Meiboom, S., and Gill, D. (1958). Modified spin-echo method for measuring nuclear relaxation times. Rev. Sci. Instrum. 29, 688-691. doi: 10.1063/1.1716296

Mueller, D. M., Allenspach, M., Othman, A., Saely, C. H., Muendlein, A., Vonbank, A., et al. (2015). Plasma levels of trimethylamine- N-oxide are confounded by impaired kidney function and poor metabolic control. Atherosclerosis 243, 638-644. doi: 10.1016/j.atherosclerosis.2015.10.091

Nicholson, J. K., Foxall, P. J., Spraul, M., Farrant, R. D., and Lindon, J. C. (1995). $750 \mathrm{MHz} 1 \mathrm{H}$ and $1 \mathrm{H}-13 \mathrm{C}$ NMR spectroscopy of human blood plasma. Anal. Chem. 67, 793-811. doi: 10.1021/ac00101a004

Nicholson, J. K., and Lindon, J. C. (2008). Systems biology: metabonomics. Nature 455, 1054-1056.

Nomenclature and criteria for diagnosis of ischemic heart disease (1979). Report of the Joint international society and federation of cardiology/World Health Organization task force on standardization of clinical nomenclature. Circulation 59, 606-609

Oliver, O., and Martin, M. (2010). Choline in acute coronary syndrome: an emerging biomarker with implications for the integrated assessment of

\section{ACKNOWLEDGMENTS}

We thank Jian-Hua Huang (Hunan Academy of Chinese Medicine) for useful discussions and suggestions.

\section{SUPPLEMENTARY MATERIAL}

The Supplementary Material for this article can be found online at: https://www.frontiersin.org/articles/10.3389/fphys. 2019.01109/full\#supplementary-material

plaque vulnerability. Expert Rev. Mol. Diagn. 10, 159-171. doi: 10.1586/ erm.10.2

Rowland, A., Miners, J. O., and Mackenzie, P. I. (2013). The UDPglucuronosyltransferases: their role in drug metabolism and detoxification. Int. J. Biochem. Cell Biol. 45, 1121-1132. doi: 10.1016/j.biocel.2013.02.019

Rubin, G. D. (2013). Emerging and evolving roles for CT in screening for coronary heart disease. J. Am. Coll. Radiol. 10, 943-948. doi: 10.1016/j.jacr.2013. 09.018

Sabatine, M. S., Liu, E., Morrow, D. A., Heller, E., McCarroll, R., Wiegand, R., et al. (2005). Metabolomic identification of novel biomarkers of myocardial ischemia. Circulation 112, 3868-3875. doi: 10.1161/circulationaha.105.569137

Steenge, G. R., Verhoef, P., and Katan, M. B. (2003). Betaine supplementation lowers plasma homocysteine in healthy men and women. J. Nutr. 133, 12911295. doi: $10.1093 /$ jn/133.5.1291

Subcommittee of Cardiovascular Diseases of China Society of Integrated Traditional Chinese, and Western Medicine (1991). Criteria for TCM syndrome differentiation of patients with coronary heart disease. Chin. J. Integr. Trad. West Med. 11, 257-258. doi: 10.1155/2018/7356546

Sun, S. J., Dai, J. Y., Wang, W. Y., Cao, H. J., Fang, J. W., Hu, Y. Y., et al. (2012). Metabonomic evaluation of ZHENG differentiation and treatment by fuzhenghuayu tablet in hepatitis-b-caused cirrhosis. Evid. Based Complement. Alternat. Med. 2012:453503. doi: 10.1155/2012/453503

Ueland, P. M. (2011). Choline and betaine in health and disease. J. Inherit. Metab. Dis. 2011, 3-15. doi: 10.1007/s10545-010-9088-4

Wang, M., Lamers, R. J., Korthout, H. A., van Nesselrooij, J. H., Witkamp, R. F., van der Heijden, R., et al. (2005). Metabolomics in the context of systems biology: bridging traditional Chinese medicine and molecular pharmacology. Phytother Res. 19, 173-182. doi: 10.1002/ptr.1624

Wei, X., Wan, L., Liu, S. M., Xia, J. D., Yi, L. Z., and Wang, D. S. (2013). Study on Plasma Metabolism of coronary heart disease with blood stasis syndrome. J. Tradit. Chin. Med. 54, 587-591.

$\mathrm{Xu}, \mathrm{H}$., and Chen, K. J. (2008). Integrative medicine: the experience from China. J. Altern. Complement. Med. 14, 3-7. doi: 10.1089/acm.2006.6329

Zhao, H. H., Wang, W., and Guo, S. Z. (2008). Proteomics Study on Unstable Angina Pectoris Patients with Blood Stasis Syndrome. Chin. J. Arterioscler. 16, 545-548.

Zhao, L. L., Wan, L., Qiu, X., Li, R. M., and Wang, D. S. (2014). A metabonomics profiling study on phlegm syndrome and blood-stasis syndrome in coronary heart disease patients using liquid chromatography/quadrupole time-of-flight mass spectrometry. Evid. Based Complement. Alternat. Med. 2014:385102. doi: $10.1155 / 2014 / 385102$

Conflict of Interest Statement: The authors declare that the research was conducted in the absence of any commercial or financial relationships that could be construed as a potential conflict of interest.

Copyright (c) 2019 Zhao, Qiu, Wang, Li and Wang. This is an open-access article distributed under the terms of the Creative Commons Attribution License (CC BY). The use, distribution or reproduction in other forums is permitted, provided the original author(s) and the copyright owner(s) are credited and that the original publication in this journal is cited, in accordance with accepted academic practice. No use, distribution or reproduction is permitted which does not comply with these terms. 